\title{
Verzeichnis der Abkürzungen
}

\begin{tabular}{|c|c|c|c|}
\hline a & auch & ArbGG & Arbeitsgerichtsgesetz idF der \\
\hline $\mathrm{aA}$ & andere(r) Ansicht & & Bekanntmachung vom 2.7. \\
\hline $\mathrm{aaO}$ & am angegebenen Ort & & 1979, BGBI. I S 853, 1036 \\
\hline $\mathrm{Abl}$ & Amtsblatt & Art & Artikel \\
\hline Abs & Absatz & ARUG & Gesetz zur Umsetzung der \\
\hline Abt & Abteilung & & Richtlinie $2007 / 36 /$ EG vom \\
\hline Abw & abweichend & & 11.7.2007 über die Ausübung \\
\hline AcP & Archiv für civilistische Praxis & & bestimmter Rechte von Ak- \\
\hline \multirow[t]{2}{*}{ ADHGB } & $\begin{array}{l}\text { Allgemeines Deutsches Han- } \\
\text { delsgesetzbuch v } 1861 \mathrm{idF} \\
\text { der Aktienrechtsnovelle }\end{array}$ & & $\begin{array}{l}\text { tionären in börsennotierten } \\
\text { Gesellschaften (ARUG), BGBI } \\
2009 \text { I Nr 50. } 2479\end{array}$ \\
\hline & v 18.7.1884 & Aufl & Auflage \\
\hline $\mathrm{aE}$ & am Ende & & \\
\hline \multirow[t]{3}{*}{ AEUV } & $\begin{array}{l}\text { Vertrag über die Arbeitswei- } \\
\text { se der Europäischen Union }\end{array}$ & BaFin & $\begin{array}{l}\text { Bundesanstalt für Finanz- } \\
\text { dienstleistungsaufsicht }\end{array}$ \\
\hline & idF mit Geltung vom 1.12. & BAG & Bundesarbeitsgericht \\
\hline & $2009, A B I 2008$ Nr C 115/1, & BAnz & Bundesanzeiger \\
\hline $\mathrm{aF}$ & $\begin{array}{l}\text { ber. ABI } 2009 \text { Nr C 290/1 } \\
\text { alte(r) Fassung }\end{array}$ & BayObLG & $\begin{array}{l}\text { Bayerisches Oberstes Lan- } \\
\text { desgericht }\end{array}$ \\
\hline AG & $\begin{array}{l}\text { Aktiengesellschaft, Die Akti- } \\
\text { engesellschaft (Zeitschrift) }\end{array}$ & BayVBI & $\begin{array}{l}\text { Bayerische Verwaltungs- } \\
\text { blätter }\end{array}$ \\
\hline \multirow[t]{2}{*}{ AGG } & $\begin{array}{l}\text { Allgemeines Gleichbehand- } \\
\text { lungsgesetz vom 14. August }\end{array}$ & BayVGH & $\begin{array}{l}\text { Bayerischer Verwaltungs- } \\
\text { gerichtshof }\end{array}$ \\
\hline & 2006 (BGBI. I S 1897) & BayVwVfG & Bayerisches Verwaltungsver- \\
\hline \multirow[t]{2}{*}{ AktG } & Aktiengesetz idF v 6.9.1965, & & fahrensgesetz v 23.12.1976 \\
\hline & BGBI. I S 1089 & BB & Betriebsberater (Zeitschrift) \\
\hline AktG 1937 & $\begin{array}{l}\text { Aktiengesetz idF v 30.1.1937, } \\
\text { RGBI. I S } 107\end{array}$ & BBergG & $\begin{array}{l}\text { Bundesberggesetz vom } \\
\text { 13.8.1980, BGBI. I S } 1310\end{array}$ \\
\hline allg & allgemein & $\mathrm{Bd}$ & Band \\
\hline Alt & Alternative & Bearb & Bearbeiter, Bearbeitung \\
\hline a.M. & am Main & bearb & bearbeitet \\
\hline \multirow[t]{5}{*}{ AnfG } & Gesetz über die Anfechtung & begr & begründet \\
\hline & von Rechtshandlungen eines & Bek & Bekanntmachung \\
\hline & Schuldners außerhalb des & Bekl & Beklagter \\
\hline & Insolvenzverfahrens idF v & ber & berichtigt \\
\hline & 5.10.1994, BGBI. I S 2911 & betr & betreffend \\
\hline Anh & Anhang & BetrAVG & Gesetz zur Verbesserung der \\
\hline Anm & Anmerkung & & betrieblichen Altersversor- \\
\hline \multirow[t]{3}{*}{ AO } & Abgabenordnung v 16.3.1976 & & gung (Betriebsrentengesetz) \\
\hline & idF d Bekanntmachung vom & & vom 19. Dezember 1974, \\
\hline & 1.10.2002, BGBI. I S 3866 & & BGBI. I S 3610 \\
\hline \multirow[t]{3}{*}{ ApoG } & Apothekengesetz idF der & BetrVG & Betriebsverfassungsgesetz \\
\hline & Bekanntmachung vom & & idF der Bekanntmachung v \\
\hline & 15.10.1980, BGBI. I S 1993 & & 25.9.2001, BGBI. I S 2518 \\
\hline
\end{tabular}




\begin{tabular}{|c|c|c|c|}
\hline BetrVG 1952 & $\begin{array}{l}\text { Betriebsverfassungsgesetz } \\
\text { v } 11.10 .1952\end{array}$ & $\begin{array}{l}\text { BR-Drucks } \\
\text { BRRD-Umset- }\end{array}$ & Bundesratsdrucksache \\
\hline $\mathrm{BFH}$ & Bundesfinanzhof & zungsgesetz & Gesetz zur Umsetzung der \\
\hline BGB & $\begin{array}{l}\text { Bürgerliches Gesetzbuch idF } \\
\text { der Bekanntmachung vom } \\
\text { 2.1.2002, BGBI. I S 42, 2909; } \\
\text { 2003 I S } 738\end{array}$ & & $\begin{array}{l}\text { Richtlinie } 2014 / 59 / \text { EU vom } \\
\text { 15. Mai } 2014 \text { zur Festlegung } \\
\text { des Rahmens für die Sanie- } \\
\text { rung und Abwicklung von }\end{array}$ \\
\hline $\mathrm{BGBI}$ & Bundesgesetzblatt & & Kreditinstituten und Wertpa- \\
\hline $\mathrm{BGH}$ & Bundesgerichtshof & & pierfirmen (Bank Recovery \\
\hline BGHSt & $\begin{array}{l}\text { Entscheidungen des Bun- } \\
\text { desgerichtshofs in Straf- } \\
\text { sachen }\end{array}$ & & $\begin{array}{l}\text { and Resolution Directive) } \\
\text { (BRRD-Umsetzungsgesetz) } \\
\text { vom 10. Dezember 2014, }\end{array}$ \\
\hline $\mathrm{BGHZ}$ & $\begin{array}{l}\text { Entscheidungen des Bun- } \\
\text { desgerichtshofs in Zivil- } \\
\text { sachen }\end{array}$ & BSG & $\begin{array}{l}\text { BGBI } 2014 \text { Teil I Nr } 59 \text { vom } \\
\text { 18. Dezember 2014, S } 2091 \\
\text { Bundessozialgericht }\end{array}$ \\
\hline BilKoG & $\begin{array}{l}\text { Gesetz zur Kontrolle von } \\
\text { Unternehmensabschlüssen } \\
\text { v 15.12.2004, BGBI I } 3408\end{array}$ & $\begin{array}{l}\text { BT-Drucks } \\
\text { BV }\end{array}$ & $\begin{array}{l}\text { Bundestagsdrucksache } \\
\text { Bayerische Verfassung idF } \\
\text { der Bekanntmachung } v \text { 15.12. }\end{array}$ \\
\hline BilReG & $\begin{array}{l}\text { Bilanzreformgesetz v } \\
4.12 .04, \mathrm{BGBI} I 3166\end{array}$ & BVerfG & $\begin{array}{l}\text { 1998, GVBI S } 991 \\
\text { Bundesverfassungsgericht }\end{array}$ \\
\hline BilRUG & $\begin{array}{l}\text { Bilanzrichtlinie- } \\
\text { Umsetzungsgesetz vom } \\
\text { 22.7.2015, BGBI I, } 1245\end{array}$ & BVerfGE & $\begin{array}{l}\text { Entscheidungen des Bun- } \\
\text { desverfassungsgerichts } \\
\text { Bundeszentralregistergesetz }\end{array}$ \\
\hline BiRiLiG & $\begin{array}{l}\text { Gesetz zur Durchführung der } \\
\text { Vierten, Siebenten und Ach- } \\
\text { ten Richtlinie des Rates der } \\
\text { Europäischen Gemeinschaf- } \\
\text { ten zur Koordinierung des }\end{array}$ & bzw & $\begin{array}{l}\text { idF der Bekanntmachung } \\
\text { vom 21.9.1984, BGBI. I } \\
\text { S 1229, 1985 I S } 195 \\
\text { beziehungsweise }\end{array}$ \\
\hline & $\begin{array}{l}\text { Gesellschaftsrechts (Bilanz- } \\
\text { richtlinien-Gesetz) v } 19.12 \text {. } \\
\text { 1985, BGBI I, S } 2355\end{array}$ & $\begin{array}{l}\text { CDO } \\
\text { CDS } \\
\text { CEO }\end{array}$ & $\begin{array}{l}\text { Credit Default Option } \\
\text { Credit Default Swap } \\
\text { Chief Executive Officer }\end{array}$ \\
\hline BKR & $\begin{array}{l}\text { Zeitschrift für Bank- und } \\
\text { Kapitalmarktrecht }\end{array}$ & CESR & $\begin{array}{l}\text { Committee of European } \\
\text { Securities Regulators - Aus- }\end{array}$ \\
\hline BMF & $\begin{array}{l}\text { Bundesministerium der } \\
\text { Finanzen }\end{array}$ & & $\begin{array}{l}\text { schuss der Europäischen } \\
\text { Wertpapierregulierungsbe- }\end{array}$ \\
\hline BMJV & $\begin{array}{l}\text { Bundesministerium der } \\
\text { Justiz und für Verbraucher- } \\
\text { schutz }\end{array}$ & $\begin{array}{l}\text { cic } \\
\text { coVInsAG }\end{array}$ & $\begin{array}{l}\text { hörden } \\
\text { culpa in contrahendo } \\
\text { COVID-19-Insolvenzausset- }\end{array}$ \\
\hline BNotO & $\begin{array}{l}\text { Bundesnotarordnung } \\
\text { v 13.2.1937 }\end{array}$ & & $\begin{array}{l}\text { zungsgesetz = Art. } 1 \text { COVID- } \\
\text { 19-Gesetz vom 27.3.2020, }\end{array}$ \\
\hline BörsG & $\begin{array}{l}\text { Börsengesetz v 16.7.2007, } \\
\text { BGBI. I S 1330, } 1351\end{array}$ & CSDR & $\begin{array}{l}\text { BGBI. I, } 569 \\
\text { Central Securities Depositary }\end{array}$ \\
\hline BörsZulV & $\begin{array}{l}\text { Börsenzulassungs- } \\
\text { verordnung }\end{array}$ & & Regulation \\
\hline BR & Bundesrat & DAV & Deutscher Anwaltsverein \\
\hline BRAO & $\begin{array}{l}\text { Bundesrechtsanwaltsord- } \\
\text { nung } v 1.8 .1959\end{array}$ & $\begin{array}{l}\mathrm{DAX} \\
\mathrm{DB}\end{array}$ & $\begin{array}{l}\text { Deutscher Aktienindex } \\
\text { Der Betrieb (Zeitschrift) }\end{array}$ \\
\hline
\end{tabular}




\begin{tabular}{|c|c|c|c|}
\hline DCGK & $\begin{array}{l}\text { Deutscher Corporate Gover- } \\
\text { nance Kodex }\end{array}$ & $\begin{array}{l}\text { EuGH } \\
\text { EulnsVo }\end{array}$ & $\begin{array}{l}\text { Europäischer Gerichtshof } \\
\text { Verordnung }(\mathrm{EG}) \mathrm{Nr} 1346 /\end{array}$ \\
\hline DepotG & Depotgesetz idF der Bek & & 2000 des Rates vom 29. Mai \\
\hline ders & $\begin{array}{l}\text { v 11.1.1995, BGBI. I S } 34 \\
\text { derselbe }\end{array}$ & & $\begin{array}{l}2000 \text { über Insolvenzver- } \\
\text { fahren }\end{array}$ \\
\hline $\mathrm{dh}$ & das heißt & EUV & Vertrag über die Europäische \\
\hline dies & dieselbe & & Union idF mit Geltung \\
\hline DNotZ & Deutsche Notar-Zeitschrift & & vom 1.12.2009, ABI 2008 \\
\hline DPR & $\begin{array}{l}\text { Deutsche Prüfstelle für } \\
\text { Rechnungslegung e.V. }\end{array}$ & & $\begin{array}{l}\text { Nr C 115/1, ber. ABI } 2009 \\
\text { Nr C 290/1 }\end{array}$ \\
\hline DrittelbG & $\begin{array}{l}\text { Drittelbeteiligungsgesetz } \\
\text { vom 18.5.2004, BGBl. I S } 974\end{array}$ & EuZW & $\begin{array}{l}\text { Europäische Zeitschrift für } \\
\text { Wirtschaftsrecht }\end{array}$ \\
\hline DStR & $\begin{array}{l}\text { Deutsches Steuerrecht (Zeit- } \\
\text { schrift) }\end{array}$ & EWG & $\begin{array}{l}\text { Europäische Wirtschaftsge- } \\
\text { meinschaft }\end{array}$ \\
\hline DZWIR & $\begin{array}{l}\text { Deutsche Zeitschrift für Wirt- } \\
\text { schafts- und Insolvenzrecht }\end{array}$ & EWiR & $\begin{array}{l}\text { Entscheidungen zum Wirt- } \\
\text { schaftsrecht (Zeitschrift) }\end{array}$ \\
\hline$E$ & Entwurf & EWIV & $\begin{array}{l}\text { Europäische Wirtschaftliche } \\
\text { Interessenvereinigung }\end{array}$ \\
\hline $\begin{array}{l}\text { EG } \\
\text { EGBGB }\end{array}$ & $\begin{array}{l}\text { Europäische Gemeinschaft } \\
\text { Einführungsgesetz zum Bür- }\end{array}$ & EWR & $\begin{array}{l}\text { Europäischer Wirtschafts- } \\
\text { raum }\end{array}$ \\
\hline & $\begin{array}{l}\text { gerlichen Gesetzbuche idF } \\
\text { der Bekanntmachung vom } \\
\text { 21.9.1994, BGBI. I S 2494; } \\
\text { 1997 I S } 1061\end{array}$ & EWS & $\begin{array}{l}\text { Europäisches Wirtschafts- } \\
\text { und Steuerrecht (Zeitschrift) }\end{array}$ \\
\hline EGHGB & $\begin{array}{l}\text { Einführungsgesetz zum Han- } \\
\text { delsgesetzbuch v 10.5.1897, } \\
\text { RGBI S } 437\end{array}$ & FamFG & $\begin{array}{l}\text { Gesetz über das Verfahren } \\
\text { in Familiensachen und in } \\
\text { den Angelegenheiten der }\end{array}$ \\
\hline EGInsO & $\begin{array}{l}\text { Einführungsgesetz zur Insol- } \\
\text { venzordnung vom 5.10.1994, } \\
\text { BGBI.I S } 2911\end{array}$ & & $\begin{array}{l}\text { freiwilligen Gerichtsbarkeit } \\
\text { vom 17.12.2008, BGBI I, } \\
\text { S } 2586\end{array}$ \\
\hline EGV & Vertrag zur Gründung der & FAQ & frequently asked questions \\
\hline & $\begin{array}{l}\text { Europäischen Gemeinschaft } \\
\text { idF v 1.1.1995 }\end{array}$ & FAZ & $\begin{array}{l}\text { Frankfurter Allgemeine Zei- } \\
\text { tung }\end{array}$ \\
\hline EHUG & Gesetz über elektronische & FE & Fondatio Europaea \\
\hline & $\begin{array}{l}\text { Handelsregister und Ge- } \\
\text { nossenschaftsregister } \\
\text { sowie das Unternehmens- } \\
\text { register vom 10.11.2006, } \\
\text { BGBII S } 2553\end{array}$ & FGG & $\begin{array}{l}\text { Gesetz über die Angelegen- } \\
\text { heiten der freiwilligen Ge- } \\
\text { richtsbarkeit v } 17.5 .1898 \text {, } \\
\text { aufgehoben und ersetzt } \\
\text { durch das FamFG }\end{array}$ \\
\hline Einl & Einleitung & FinDAG & Gesetz über die Bundes- \\
\hline EK & Eigenkapital & & anstalt für Finanzdienst- \\
\hline e $P$ & Europäisches Parlament & & leistungsaufsicht vom \\
\hline EStG & Einkommensteuergesetz idF & & 22.4.2002, BGBII S 1310 \\
\hline & der Bekanntmachung vom & FMStG & Gesetz zur Umsetzung eines \\
\hline & $\begin{array}{l}\text { 19.10.2002, BGBI. I S 4210; } \\
\text { 2003 I S } 179\end{array}$ & & $\begin{array}{l}\text { Maßnahmenpakets zur Sta- } \\
\text { bilisierung des Finanzmark- }\end{array}$ \\
\hline EU & Europäische Union & & tes (Finanzmarktstabilisie- \\
\hline
\end{tabular}




\begin{tabular}{|c|c|c|c|}
\hline & rungsgesetz - FMStG) & hrsg & herausgegeben \\
\hline & v 17.10.2008, BGBII S 1982 & $\mathrm{HRV}$ & Handelsregisterverordnung \\
\hline $\mathrm{Fn}$ & Fußnote & Hs & Halbsatz \\
\hline \multirow[t]{2}{*}{ FRUG } & Finanzmarktrichtlinieumset- & HV & Hauptversammlung \\
\hline & $\begin{array}{l}\text { zungsgsetz v } 16.7 .2007 \\
\text { BGBI I, } 1330\end{array}$ & IAS & $\begin{array}{l}\text { International Accounting } \\
\text { Standards }\end{array}$ \\
\hline FS & Festschrift & IASB & International Accounting \\
\hline \multirow[t]{2}{*}{ FWB } & Frankfurter Wertpapierbörse & & Standards Board \\
\hline & & $\mathrm{idF}$ & in der Fassung \\
\hline \multirow[t]{2}{*}{ GAAP } & Generally Accepted Account- & $\mathrm{iE}$ & im Einzelnen \\
\hline & ing Principles & IFRS & International Financial \\
\hline \multirow[t]{2}{*}{ GemSObGerB } & Gemeinsamer Senat der ober- & & Reporting Standards \\
\hline & sten Gerichtshöfe des Bundes & iG & in Gründung \\
\hline \multirow[t]{2}{*}{ GenG } & $\begin{array}{l}\text { Genossenschaftsgesetz idF } \\
\text { vom 16.10.2006, BGBl. I }\end{array}$ & InsO & $\begin{array}{l}\text { Insolvenzordnung v. } \\
5.10 .1994\end{array}$ \\
\hline & S 2230 & InvG & Investmentgesetz vom \\
\hline \multirow[t]{3}{*}{ GewStG } & Gewerbesteuergesetz idF der & & 15.12.2003, BGBI. I S 2676 \\
\hline & Bekanntmachung vom & IPR & Internationales Privatrecht \\
\hline & 15.10.2002, BGBI. I S 4167 & IPRax & Praxis des Internationalen \\
\hline \multirow[t]{2}{*}{ GG } & $\begin{array}{l}\text { Grundgesetz für die Bundes- } \\
\text { republik Deutschland }\end{array}$ & is & $\begin{array}{l}\text { Privat- und Verfahrensrechts } \\
\text { im Sinne }\end{array}$ \\
\hline & v 23.5.1949 & iSd & im Sinne des \\
\hline \multirow[t]{2}{*}{$\mathrm{GmbH}$} & Gesellschaft(en) mit be- & iSv & im Sinne von \\
\hline & schränkter Haftung & iü & im übrigen \\
\hline \multirow[t]{2}{*}{ GmbHG } & Gesetz betreffend die Ge- & $\mathrm{iVm}$ & in Verbindung mit \\
\hline & $\begin{array}{l}\text { sellschaften mit beschränk- } \\
\text { ter Haftung } v 20.4 .1892\end{array}$ & IWRZ & $\begin{array}{l}\text { Zeitschrift für Internationa- } \\
\text { les Wirtschaftsrecht }\end{array}$ \\
\hline GmbHR & $\begin{array}{l}\text { GmbH-Rundschau (Zeit- } \\
\text { schrift) }\end{array}$ & $\mathrm{JBI}$ & Juristische Blätter \\
\hline \multirow[t]{2}{*}{ GoB } & Grundsätze ordnungsgemä- & JuS & Juristische Schulung \\
\hline & Ber Geschäftsführung & JW & Juristische Wochenschrift \\
\hline GS & Gedächtnisschrift & $\mathrm{JZ}$ & Juristenzeitung \\
\hline \multirow[t]{2}{*}{ GVBI } & Gesetz- und Verordnungs- & & \\
\hline & blatt & KAGB & Kapitalanlagegesetzbuch \\
\hline \multirow[t]{2}{*}{ GVG } & Gerichtsverfassungsgesetz & & vom 4.7.2013, BGBI I, 1981 \\
\hline & $\begin{array}{l}\text { v 27.1.1877 idF der Bek v } \\
\text { 9.5.1975 }\end{array}$ & KAGG & $\begin{array}{l}\text { Gesetz über Kapitalanlage- } \\
\text { gesellschaften idF der Be- }\end{array}$ \\
\hline \multirow[t]{3}{*}{ GWB } & Gesetz gegen Wettbewerbs- & & kanntmachung vom \\
\hline & beschränkungen idF der & & 9.9.1998, BGBI. I S 2726 \\
\hline & $\begin{array}{l}\text { Bekanntmachung vom } \\
\text { 15.7.2005, BGBI. I S } 2114\end{array}$ & KapAEG & $\begin{array}{l}\text { Kapitalaufnahmeerleichte- } \\
\text { rungsgesetz vom 20.4.1998, } \\
\text { BGBI. I S } 707\end{array}$ \\
\hline $\mathrm{HGB}$ & $\begin{array}{l}\text { Handelsgesetzbuch v } \\
10.5 .1897\end{array}$ & KapMuG & $\begin{array}{l}\text { Kapitalanleger-Musterver- } \\
\text { fahrensgesetz vom } 16.8 \text {. }\end{array}$ \\
\hline HM & herrschende Meinung & & 2005, BGBII S 2437 \\
\hline HRefG & $\begin{array}{l}\text { Handelsrechtsreformgesetz } \\
\text { vom 22.6.1998, BGBI. IS } 1474\end{array}$ & KG & $\begin{array}{l}\text { Kammergericht, Kommandit- } \\
\text { gesellschaft(en) }\end{array}$ \\
\hline
\end{tabular}




\begin{tabular}{|c|c|c|c|}
\hline KGaA & $\begin{array}{l}\text { Kommanditgesellschaft auf } \\
\text { Aktien }\end{array}$ & MaßnG-GesR & $\begin{array}{l}\text { Gesetz über Maßnahmen im } \\
\text { Gesellschaftsrecht ... zur }\end{array}$ \\
\hline $\mathrm{Kl}$ & Kläger & & Bekämpfung der Auswirkun- \\
\hline \multirow[t]{2}{*}{ KIAnISchG } & Kleinanlegerschutzgesetz & & gen der COVID-19-Pandemie \\
\hline & vom 3.7.2015, BGBI I, 1114 & & $=$ Art. 2 des COVID-19-G vom \\
\hline KO & $\begin{array}{l}\text { Konkursordnung v 10.2.1877 } \\
\text { idF der Bek v 20.5.1898, } \\
\text { RGBI S } 612\end{array}$ & $\mathrm{MbB}$ & $\begin{array}{l}\text { 27. 3. 2020, BGBI. I, } 569 \\
\text { (Partnerschaft) mit be- } \\
\text { schränkter Berufshaftung }\end{array}$ \\
\hline Kom & Kommission, Kommentar & MgVG & Gesetz zur Umsetzung der \\
\hline KonTraG & $\begin{array}{l}\text { Gesetz zur Kontrolle und } \\
\text { Transparenz im Unterneh- } \\
\text { mensbereich vom 27.4.1998, } \\
\text { BGBII S } 786 .\end{array}$ & & $\begin{array}{l}\text { Regelungen über die Mitbe- } \\
\text { stimmung der Arbeitnehmer } \\
\text { bei einer Verschmelzung von } \\
\text { Kapitalgesellschaften aus }\end{array}$ \\
\hline KostO & $\begin{array}{l}\text { Kostenordnung idF der Be- } \\
\text { kanntmachung vom } 26.7 \text {. } \\
\text { 1957, BGBl. I S } 960\end{array}$ & & $\begin{array}{l}\text { verschiedenen Mitgliedstaa- } \\
\text { ten vom 21.12.2006, BGBII } \\
\text { S } 3332 \text {. }\end{array}$ \\
\hline KStG & $\begin{array}{l}\text { Körperschaftsteuergesetz } \\
\text { idF der Bekanntmachung } \\
\text { v 15.10.2002, BGBI. I S } 4144\end{array}$ & MicroBilg & $\begin{array}{l}\text { Kleinstkapitalgesellschaf- } \\
\text { ten-Bilanzrechtsänderungs- } \\
\text { gesetz vom 20.12.2012, }\end{array}$ \\
\hline \multirow[t]{4}{*}{ KWG } & Kreditwesengesetz idF der & & BGBI I, 2751 \\
\hline & $\begin{array}{l}\text { Bekanntmachung vom 9.9. } \\
\text { 1998, BGBI.IS } 2776 \text { zuletzt } \\
\text { geändert durch Gesetz zur } \\
\text { Umsetzung der Transparenz- }\end{array}$ & MiFiD II & $\begin{array}{l}\text { Markets in Financial Instru- } \\
\text { ments Directive II (Richtlinie } \\
\text { 2014/65/EU, ABI } 2014 \text { L 173, } \\
\text { 349) }\end{array}$ \\
\hline & $\begin{array}{l}\text { richtlinie-Änderungs- } \\
\text { richtlinie vom 20. November } \\
2015, \text { BGBI.IS } 2029 \text {. }\end{array}$ & MiFiR & $\begin{array}{l}\text { Regulation (Verordnung } \\
\mathrm{Nr} 600 / 2014, A B \mid 2014 \text { L 173, } \\
\text { 84) }\end{array}$ \\
\hline & & MitbestErgG & Gesetz zur Ergänzung des \\
\hline LAG & Landesarbeitsgericht & & Gesetzes über die Mitbe- \\
\hline LG & Landgericht & & stimmung der Arbeitnehmer \\
\hline li Sp & linke Spalte & & in den Aufsichtsräten und Vor- \\
\hline lit & litera & & ständen der Unternehmen \\
\hline \multirow[t]{2}{*}{ LöschG } & $\begin{array}{l}\text { Gesetz über die Auflösung } \\
\text { und Löschung von Gesell- } \\
\text { schaften und Genossen- }\end{array}$ & & $\begin{array}{l}\text { des Bergbaus und der Eisen } \\
\text { und Stahl erzeugenden Indus- } \\
\text { triev7.8.1956, BGBIIS707 }\end{array}$ \\
\hline & schaften v 9.10 .1934 & MitbestG & Mitbestimmungsgesetz vom \\
\hline LPG & $\begin{array}{l}\text { Landwirtschaftliche Produk- } \\
\text { tionsgenossenschaft }\end{array}$ & $\mathrm{mN}$ & $\begin{array}{l}\text { 4.5.1976, BGBI. I S } 1153 \\
\text { mit Nachweis(en) }\end{array}$ \\
\hline \multirow[t]{2}{*}{ LSG } & Landessozialgericht & mwN & mit weiteren Nachweisen \\
\hline & & MoMiG & Gesetz zur Modernisierung \\
\hline MaKonV & $\begin{array}{l}\text { Marktmanipulationskonkre- } \\
\text { tisierungsverordnung vom } \\
\text { 1.3.2005, BGBI I S } 515\end{array}$ & & $\begin{array}{l}\text { des GmbH-Rechts und zur } \\
\text { Bekämpfung von Missbräu- } \\
\text { chen v 23.10.2008, BGBII }\end{array}$ \\
\hline \multirow[t]{2}{*}{ MAR } & MarketAbuseRegulation & & S 2026 \\
\hline & $\begin{array}{l}\text { (Marktmissbrauchsverord- } \\
\text { nung Nr 596/2014, ABI } 2014 \\
\text { L 173, 1) }\end{array}$ & $\begin{array}{l}\text { Montan } \\
\text { mitbestG }\end{array}$ & $\begin{array}{l}\text { Gesetz über die Mitbestim- } \\
\text { mung der Arbeitnehmer in }\end{array}$ \\
\hline
\end{tabular}




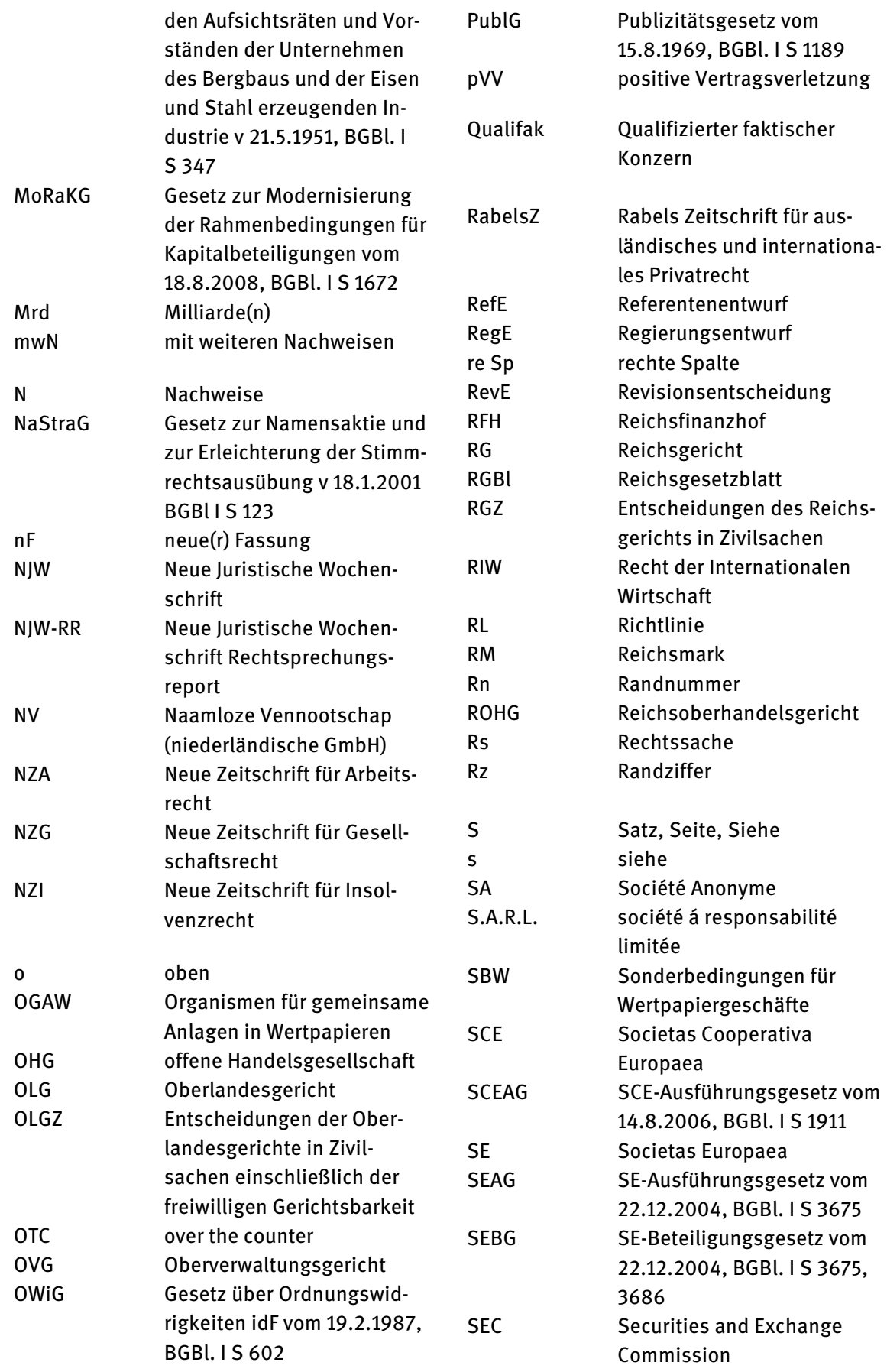




\begin{tabular}{|c|c|c|c|}
\hline \multirow[t]{3}{*}{ SEEG } & Gesetz zur Einführung der & UmwG & Umwandlungsgesetz vom \\
\hline & vom 22.12.2004, BGBI I & \multirow[t]{3}{*}{ UWG } & Gesetz gegen den unlauteren \\
\hline & S 3675 & & Wettbewerb vom 3.7.2004, \\
\hline SLNE & $\begin{array}{l}\text { Sociedad Limitada Nueva } \\
\text { Empresa }\end{array}$ & & BGBI. I S 1414 \\
\hline sog & sogenannte & v & von, vom \\
\hline Sp & Spalte & \multirow[t]{2}{*}{ VAG } & Versicherungsaufsichts- \\
\hline SPE & $\begin{array}{l}\text { Societas Privata Europaea, } \\
\text { Europäische Privatgesell- } \\
\text { schaft }\end{array}$ & & $\begin{array}{l}\text { gesetz idF der Bekannt- } \\
\text { machung vom 17.12.1992, } \\
\text { BGBI. I S } 2\end{array}$ \\
\hline SpruchG & $\begin{array}{l}\text { Gesetz zur Neuordnung des } \\
\text { gesellschaftsrechtlichen } \\
\text { Spruchverfahrens vom } \\
\text { 12.6.2003, BGBI I S } 838 \text {. }\end{array}$ & VerkPG & $\begin{array}{l}\text { Verfasser } \\
\text { Verkaufsprospektgesetz idF } \\
\text { der Bekanntmachung vom } \\
\text { 9.9.1998, BGBI. I S } 2701\end{array}$ \\
\hline StBerG & $\begin{array}{l}\text { Steuerberatungsgesetz idF- } \\
\text { der Bekanntmachung vom } \\
\text { 4.11.1975, BGBI. I S } 2735\end{array}$ & VermAnlG & $\begin{array}{l}\text { Gesetz über Vermögensan- } \\
\text { lagen vom 6.12.2011, BGBI I, } \\
2481\end{array}$ \\
\hline StGB & $\begin{array}{l}\text { Strafgesetzbuch idf der Be- } \\
\text { kanntmachung vom } \\
\text { 13.11.1998, BGBI. I S } 3322 \\
\text { streitig }\end{array}$ & $\begin{array}{l}\text { VGH } \\
\text { vgl } \\
\text { VGR }\end{array}$ & $\begin{array}{l}\text { Verwaltungsgerichtshof } \\
\text { vergleiche } \\
\text { Gesellschaftsrechtliche } \\
\text { Vereinigung }\end{array}$ \\
\hline StuW & $\begin{array}{l}\text { Steuer und Wirtschaft (Zeit- } \\
\text { schrift) }\end{array}$ & $\begin{array}{l}\text { Vo } \\
\text { Vorbem }\end{array}$ & $\begin{array}{l}\text { Verordnung } \\
\text { Vorbemerkung }\end{array}$ \\
\hline $\begin{array}{l}\text { SUP } \\
\text { SZ }\end{array}$ & $\begin{array}{l}\text { Societas Unius Personae } \\
\text { Süddeutsche Zeitung }\end{array}$ & $\begin{array}{l}\text { Vorn } \\
\text { vorst } \\
\text { VorstAG }\end{array}$ & $\begin{array}{l}\text { Vornote } \\
\text { vorstehend } \\
\text { Gesetz zur Angemessenheit }\end{array}$ \\
\hline $\begin{array}{l}\text { TOP } \\
\text { TransPuG/ }\end{array}$ & Tagesordnungspunkt & & $\begin{array}{l}\text { von Vorstandsvergütungen } \\
\text { vom 31.Juli 2009, BGBII }\end{array}$ \\
\hline TrPublG & $\begin{array}{l}\text { Transparenz- und Publizi- } \\
\text { tätsgesetz v. 19.7.2002, } \\
\text { BGBII S } 2681\end{array}$ & \multirow[t]{2}{*}{ VorstOG } & $\begin{array}{l}2009 \text { S } 2509 \\
\text { Gesetz über die Offen- } \\
\text { legung von Vorstands- }\end{array}$ \\
\hline \multirow[t]{2}{*}{ TUG } & $\begin{array}{l}\text { Transparenzrichtlinie- } \\
\text { Umsetzungsgesetz vom }\end{array}$ & & $\begin{array}{l}\text { vergütungen vom 3.8.2005, } \\
\text { BGBI I S } 2267\end{array}$ \\
\hline & 5.1.2007, BGBI. I S 10 & \multirow[t]{4}{*}{ VW-Gesetz } & $\begin{array}{l}\text { Gesetz über die Überführung } \\
\text { der Anteilsrechte an der }\end{array}$ \\
\hline u & unten & & Volkswagen Gesellschaft \\
\hline ua & unter anderem, und andere & & mit beschränkter Haftung in \\
\hline \multirow[t]{2}{*}{ UBGG } & $\begin{array}{l}\text { Gesetz über Unternehmens- } \\
\text { beteiligungsgesellschaften }\end{array}$ & & $\begin{array}{l}\text { private Hand vom 21.7.1960, } \\
\text { BGBI. I S } 585\end{array}$ \\
\hline & $\begin{array}{l}\text { vom 17.12.1986, BGBI I } \\
\text { S } 2488 .\end{array}$ & VwGO & $\begin{array}{l}\text { Verwaltungsgerichtsordnung } \\
\text { idF der Bekanntmachung }\end{array}$ \\
\hline UG & Unternehmergesellschaft & & vom 19.3.1991, BGBI. I S 686 \\
\hline UMAG & $\begin{array}{l}\text { Gesetz zur Unternehmensin- } \\
\text { tegrität und Modernisierung } \\
\text { des Anfechtungsrechts vom } \\
\text { 22.9.2005, BGBI I S } 2802\end{array}$ & VwVfG & $\begin{array}{l}\text { Verwaltungsverfahrensge- } \\
\text { setz idF der Bekanntma- } \\
\text { chung vom 23.1.2003, BGBI. } \\
\text { I S } 102\end{array}$ \\
\hline
\end{tabular}




\begin{tabular}{|c|c|c|c|}
\hline WG & Wechselgesetz v 21.6.1933 & & verfassung] v 11.8.1919, \\
\hline WKBG & Gesetz zur Förderung von & & RGBI S 1383 \\
\hline & $\begin{array}{l}\text { Wagniskapitalbeteiligungen } \\
\text { (WKBG), Art } 1 \text { des MoRaKG }\end{array}$ & WStF & $\begin{array}{l}\text { Wirtschaftsstabilisierungs- } \\
\text { fonds }\end{array}$ \\
\hline & $\begin{array}{l}\text { vom 12.8.2008 BGBI I, 1672; } \\
\text { Wagniskapitalbeteiligungs- } \\
\text { gesellschaft }\end{array}$ & WStFG & $\begin{array}{l}\text { Gesetz zur Errichtung eines } \\
\text { Wirtschaftsstabilisierungs- } \\
\text { fonds vom 27.3.2020, }\end{array}$ \\
\hline WM & Wertpapier Mitteilungen & & BGBI. I, 543 \\
\hline & (Zeitschrift) & WuB & Entscheidungssammlung \\
\hline WpAIV & $\begin{array}{l}\text { Wertpapierhandelsanzeige- } \\
\text { und Insiderverzeichnisver- } \\
\text { ordnung vom 13.12.2004, }\end{array}$ & & $\begin{array}{l}\text { zum Wirtschafts- und Bank- } \\
\text { recht }\end{array}$ \\
\hline & BGBII S 3376 & $z B$ & zum Beispiel \\
\hline WPg & $\begin{array}{l}\text { Die Wirtschaftsprüfung (Zeit- } \\
\text { schrift) }\end{array}$ & ZBB & $\begin{array}{l}\text { Zeitschrift für Bankrecht und } \\
\text { Bankwirtschaft }\end{array}$ \\
\hline WpHG & $\begin{array}{l}\text { Gesetz über den Wertpapier- } \\
\text { handel idF der Bekanntma- }\end{array}$ & ZfPW & $\begin{array}{l}\text { Zeitschrift für die gesamte } \\
\text { Privatrechtswissenschaft }\end{array}$ \\
\hline & $\begin{array}{l}\text { chung vom 9.9.1998, BGBI. I } \\
\text { S } 2708\end{array}$ & ZGR & $\begin{array}{l}\text { Zeitschrift für Unternehmens- } \\
\text { und Gesellschaftsrecht }\end{array}$ \\
\hline WpPG & $\begin{array}{l}\text { Wertpapierprospektgesetz } \\
\text { vom 22.6.2005, BGBI. I } \\
\text { S } 1698\end{array}$ & ZHR & $\begin{array}{l}\text { Zeitschrift für das gesamte } \\
\text { Unternehmens- und Gesell- } \\
\text { schaftsrecht }\end{array}$ \\
\hline WpÜG & $\begin{array}{l}\text { Wertpapiererwerbs- und } \\
\text { Übernahmegesetz vom }\end{array}$ & $\begin{array}{l}\text { Ziff } \\
\text { ZIP }\end{array}$ & $\begin{array}{l}\text { Ziffer } \\
\text { Zeitschrift für Wirtschafts- }\end{array}$ \\
\hline & 20.12.2001, BGBI. I S 3822 & & recht \\
\hline WpÜG-AV & $\begin{array}{l}\text { Verordnung über den Inhalt } \\
\text { der Angebotsunterlage, die } \\
\text { Gegenleistung bei Übernah- } \\
\text { meangeboten und Pflichtan- }\end{array}$ & ZPO & $\begin{array}{l}\text { Zivilprozessordnung idF der } \\
\text { Bekanntmachung vom } \\
5.12 .2005 \text {, BGBI. I S 3202; } \\
2006 \text { I S 431; } 2007 \text { I S } 1781\end{array}$ \\
\hline & geboten und die Befreiung & ZRP & Zeitschrift für Rechtspolitik \\
\hline & von der Verpflichtung zur & zust & zustimmend \\
\hline & $\begin{array}{l}\text { Veröffentlichung und zur } \\
\text { Abgabe eines Angebots vom }\end{array}$ & ZVG & $\begin{array}{l}\text { Gesetz über die Zwangsver- } \\
\text { steigerung und die Zwangs- }\end{array}$ \\
\hline & 27.12.2001, BGBI. I S 263 & & verwaltung vom 24.3.1897 \\
\hline WRV & $\begin{array}{l}\text { Verfassung des Deutschen } \\
\text { Reichs [Weimarer Reichs- }\end{array}$ & ZWH & $\begin{array}{l}\text { Zeitschrift für Wirtschafts- } \\
\text { strafrecht und Haftung im } \\
\text { Unternehmen }\end{array}$ \\
\hline
\end{tabular}

